\title{
Single Centre Exploration of Tubeless PCNL
}

\author{
Dr. Vipin Kumar ${ }^{*}$, Dr. Siddhant Kumar, Dr. M.K. Maheshwari, Dr. Sajad Ahmad Para
}

Dept. of General Surgery, Subharti Medical College, Delhi Meerut Bypass Road, Meerut, UP - 250005, India

DOI: $10.36347 /$ sasjs.2020.v06i03.003

| Received: 29.02.2020 | Accepted: 07.03.2020 | Published: 11.03.2020

*Corresponding author: Dr. Vipin Kumar

Abstract

Original Research Article

Introduction: The standard PCNL includes insertion of a nephrostomy tube and a Double J stent after the procedure. Tubeless PCNL i.e no neprostomy tube following a PCNL in view of reduced morbidity and hospital stay is being implanted. This leads to our study on tubeless PCNL with the objectives of evaluation of outcomes and complications with the same and to evaluate the efficacy and safety of tubeless percutaneous nephrolithotomy (PCNL). Material and methods: This study conducted in department of General Surgery, Subharti Medical College, Meerut where 100 patients were evaluated 56 of whom underwent standard PCNL and 46 underwent tubeless PCNL. In the group of tubeless PCNL, no nephrostomy was inserted after removal of the stone fragments. We compared the results of tubeless PCNL with those of the standard procedure. The operative time, blood loss, perioperative complications, postoperative analgesic requirements and duration of hospitalization in the two groups were analyzed. Conclusion: In our study we concluded that Tubeless PCNL is safe and effective methods of renal stone management and is comparable to the standard PCNL in terms of postoperative outcomes and safety and seem to have benefits in terms of length of hospitalization, postoperative pain and all these features might make tubeless PCNL the new standard.

Keywords: Centre Exploration Tubeless PCNL.

Copyright @ 2020: This is an open-access article distributed under the terms of the Creative Commons Attribution license which permits unrestricted use, distribution, and reproduction in any medium for non-commercial use (NonCommercial, or CC-BY-NC) provided the original author and source are credited.

\section{INTRODUCTION}

The etiology, treatment modalities, and morbidities of urinary stone disease are highly multifaceted. The occurrence of this condition is increasing in developed countries, and environmental factors, dietary habits, and metabolic abnormalities have a strong association [1]. Kidney stones are very common and affect at least $10 \%$ of the population. Recurrence of kidney stones has been seen in $70 \%$ of the affected patients. Various non-invasive, minimally invasive, and invasive methods have been used for the treatment of kidney stones, including medicinal treatment, extracorporeal shock wave lithotripsy (ESWL), percutaneous nephrolithotomy (PCNL), and open renal surgery. In the past 30 years, PCNL as a minimally invasive technique has proved to be an effective modality for large stones located in the kidney and upper ureter. PCNL is a more efficient treatment for stones $>2 \mathrm{~cm}$ compared with the ESWL method [2].

Percutaneous nephrolithotomy has completely changed the approach to large and complex renal calculi. This technique was first introduced in the early 1980s; PCNL decreased morbidity and minimized invasiveness, gradually replacing the open surgical approach [3].
PCNL includes four steps: access to the kidney, dilatation of the tract (access site), nephroscopy, fragmentation of stones, removal of fragments and finally inserting a nephrostomy tube at the end of the procedure. Percutaneous nephrolithotomy (PCNL) has been accepted as the primary treatment of choice for patients with a large kidney stone [4]. Conventionally, a 20-24 French nephrostomy catheter is placed usually after PCNL to offer urine drainage, prevent extravasation of urine, and make tamponade against bleeding [5, 6]. In addition, it can be used as a tract for a second-look PCNL if required. The need for insertion of a conventional large-bore nephrostomy catheter has been questioned because of its additional increase in postoperative discomfort and other morbidity, and the low incidence of second-look operations $[7,8]$.

Placement of a nephrostomy tube and/or internal ureteral stent after PCNL has been considered standard practice and it's purpose is to allow the renal puncture to heal, to provide appropriate drainage of urine, and to permit access to the collecting system, if a secondary procedure is required (9). In some patients, the placement of a nephrostomy tube and ureteral stent can be omitted. Thus, these modifications in PCNL technique allow earlier discharge from the hospital, and 
leads to more speedy recovery and also the complications of internal stenting such as ureteral paralysis and ureteral obstruction may be avoided [10].

With the development of better instrumentation and experience of PCNL, several modifications to the procedure have taken place in order to reduce the morbidity and early return to normal lifestyle. There is an ever-growing literature base documenting several technological and procedural refinements to this approach. In tubeless PCNL, the fourth step (i.e., inserting a nephrostomy tube) is not carried out. Recently, a more modern PCNL technique was introduced: totally tubeless PCNL. In this method, a nephrostomy catheter, a double $\mathrm{J}$ stent, or a ureteral catheter are not inserted at the end of procedure [11].

\section{MATERIAL AND METHODS}

This prospective study was conducted at the Surgery Department in Chhatrapati Shivaji Subharti Medical College, Meerut during period from July 2017 to February 2019. Patients chosen for this work were studied from the date of their admission to surgical wards. Patients included in the study are those who underwent PCNL. Details of history, examination and investigations of those patients were collected.

\section{STUDY DESIGN}

Type of the study: Experimental Design Study. Two groups were formed: Group 1 included patients submitted to standard (with any nephrostomy tube) and Group 2 to tubeless PCNL. Using a simple randomisation method of sealed envelopes before the procedure.

\section{SELECTION OF SUBJECT \\ Inclusion Criteria}

- $\quad$ Single stone in Pelvicalycal system. (PCS)

- Single puncture Percutaneous nephrolithotomy. (PCNL)

- Intact collecting system

\section{Exclusion Criteria}

- Patients having staghorn calculi

- Solitary kidney

- CKD kidney

- Pelvic Perforations

- Requiring multiple access

- Serious bleeding

- Previous operations or drainage (Post PCN)

\section{STUDY TOOLS}

Case reporting form

Investigations

Visual analogue scale

Patients were included in the study after obtaining informed written consent. Pre and postoperative hematocrit values, blood transfusion rates, fluoroscopy durations, post-operative complications, length of hospital stay, post-operative 1 st and $6^{\text {th }}$ hours pain scores and analgesic requirements were compared in tubeless and standard patients. The outcomes measures of interest were: (1) post-operative pain scoring and analgesia requirements; (2) duration of hospitalization and convalescence; (3) operation time; (4) major and minor complications; (5) stone-free rates.

\section{Study Protocol}

The patients fulfilling the criteria of the study were divided randomly into two groups using a simple randomisation method of sealed envelopes before the procedure (i.e Double blind study).

\section{STEPS}

- All patients received Ceftriaxone prior to surgery.

- Anaesthesia - Spinal/GA - as per the choice of anaesthetist.

- Lithotomy position is made and Cystoscopy is done using a 20Fr rigid cystoscope.

- 5Fr ureteric catheter is introduced over 0.032" guide wire for negotiation of pelvicalyceal system, pelvicalyceal system is opacified with injecting urografin dye through the ureteric catheter.

- In prone position under fluoroscopic guidance calyceal puncture is taken with the initial puncture needle (17.5Fr).

- $\quad 0.032$ " guide wire is introduced over the initial puncture needle.

- Sequential tract dilation is done with ALKEN metal dilator.

- $\quad 26 \mathrm{Fr}$ sheath used over ALKEN dilator. 18Fr nephroscope used for nephroscopy.

- $\quad$ Stone fragmented with pneumolithoclast and fragments removed.

- In standard PCNL 20Fr nephrostomy tube placed in pelvicalyceal system, while in tubeless PCNL no nephrostomy tube was used.

- Haemoglobin, urine routine and microscopy and urine culture sent on POD 1.

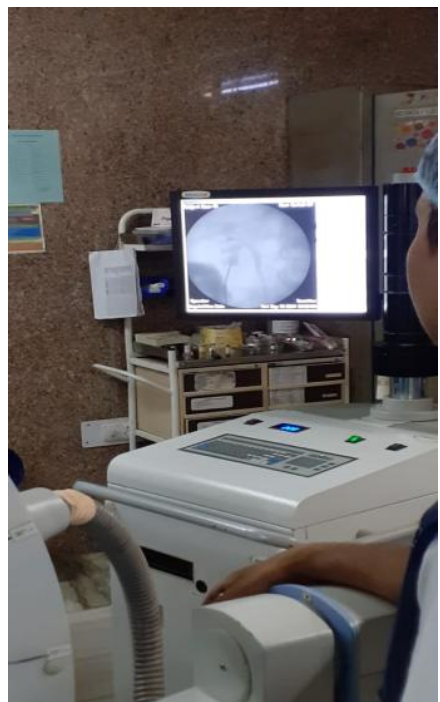

Fig-1: Guidewire inserted over initial puncture 


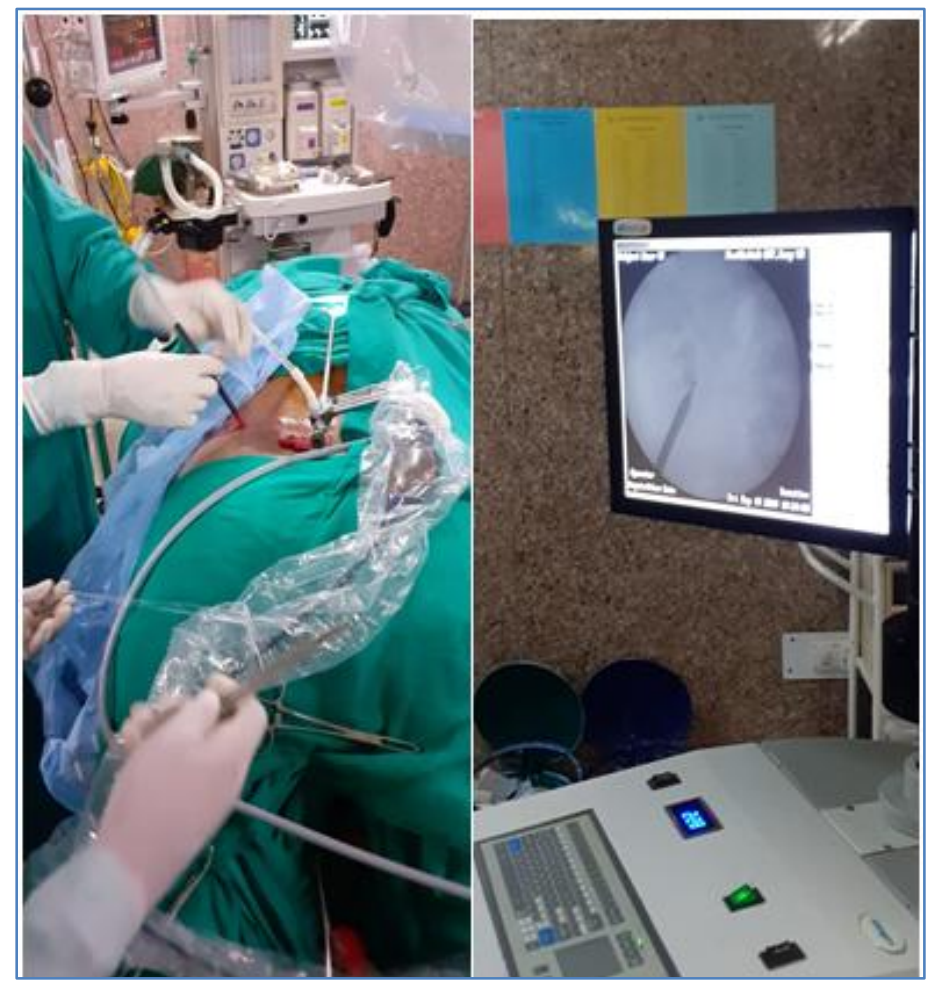

Fig-2 and 3: Sequential Dilatation

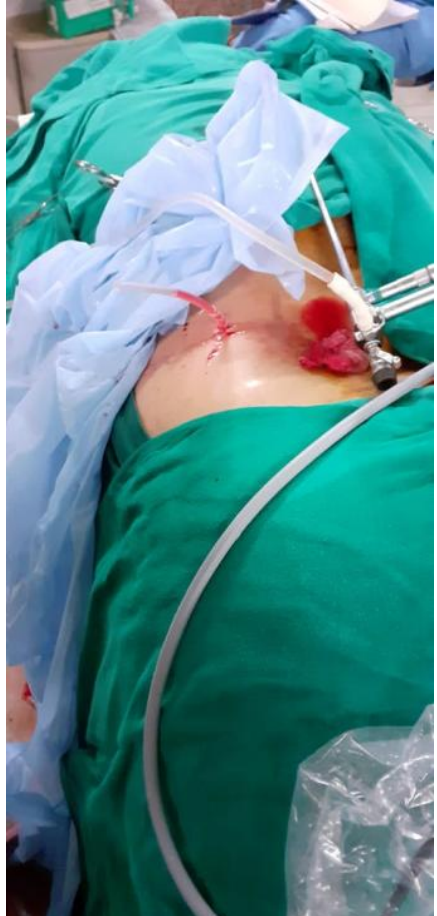

Fig-4: 20Fr Nephrostomy Placed in Standard group

\section{RESULTS AND DISCUSSION}

In our study, there were a total of 100 patients out of which 56 were in the standard group, while 44 were in the tubeless group. The highest incidence was in the standard group was seen in age group of 21-30 years and 31 to 40 years in the tubeless group and the mean age was $37.08 \pm 12.72$ in the standard group while in the tubeless group, it was $37.04 \pm 12.57$. There were a total of 24 females and 76 males. Amongst the
24 females, 10 were in the standard group and 14 in the tubeless group. Of the 76 males, 46 were in standard group, 30 were in tubeless group. In a study done by Wei-Hong, there were 659 males and 341 females. The average age was $54.4 \pm 12.6$ years [39]. Similar results in relation to sex were seen in study by Sreedhar Dayapule, which included 13 (32.5\%) females and 27 (67.5\%) males [42]. In a study by A Tyagi, 112 cases were included. In tubeless group, there were 54 cases (group A) 35 were male and 21 female patients. Male to female ratio is $1.66: 1$. The average age was 44.6 years with arrange of 20 to 65 years. In the traditional PCNL group, female ratio is $2.3: 1$ and average age is 48.8 years [69].

In our study, the common presenting complaints were right flank pain and left flank pain. Incidence of Left flank pain was 36\% overall and incidence of right flank pain was $35 \%$. In our study, left PCNL was performed in 60 patients, 31 were standard type and 29 were tubeless type. Right PCNL was done in 40 cases, 25 were standard group and 15 were of tubeless group. In study by Suresh Bhat, out of the 50 patients, 23 patients were operated for the right side (10 in standard group and 13 in the tubeless group) and 27 patients were operated for the left side (15 in standard group and 12 in the tubeless group) [70].

In our study, the mean pre-op $\mathrm{Hb}$ was $13.20 \pm$ 0.99 in the standard group and $13.20 \pm 0.79$ in the tubeless group. The mean post-operative $\mathrm{Hb}$ was 11.80 \pm 1.06 in the standard group and $12.24 \pm 0.81$ in the tubeless group. The post-operative $\mathrm{Hb}$ range was 8.614.6 in standard group and 8.8-16 in the tubeless group. 
We found that the percentage drop in hemoglobin was $17.12 \pm 8.81$ while it was only $8.08 \pm 6.65$ in the tubeless group and it was found to be statistically significant, thereby predicting less amount of blood loss in the tubeless group. Blood transfusion was required in 2 cases in the standard group, not required in any case in the tubeless group. In the standard group 2 patients required blood transfusion (one patient required 2 units and other one required 3 units). In study by Shah $\mathrm{H}$, decrease in the hemoglobin level was $0.82 \mathrm{mg} / \mathrm{dl}$ in the tubeless group while in the standard group the decrease was $0.97 \mathrm{mg} / \mathrm{dl}$; comparative analysis in this study showed the difference to be statistically insignificant [50]. Incidentally, in study by Suresh Bhat, the hemoglobin drop in the standard group was 1.01 while in the tubeless group was 1.55 , which is a contrasting evidence compared to our study [69]. In study by $\mathrm{S}$ Dayapule, drop in hemoglobin in the standard group was $0.5 \pm 0.5$ while in the tubeless group it was $0.3 \pm 0.4$ $(\mathrm{p}=0.13)$.

The total duration in the standard group was $76.89 \pm 24.29$ mins while in the tubeless group was $66.54 \pm 20.34$. The duration range in the standard group was 30-120 mins while in the tubeless group the range was 20-90 mins. Comparing both the groups in our study was, the difference in the time duration in both groups was found to be statistically significant. In study by A Tyagi, the mean duration for surgery, in the tubeless group was 56.4 mins while in the standard group it was significantly higher which 81.8 mins [69] was. In study by Shah $\mathrm{H}$, the mean time duration in the standard group was 68.8 mins while in the tubeless group was 52.2 mins with range of 22-178 mins [50]. In study by $\mathrm{S}$ Dayapule, the average duration of procedure in the tubeless group was $38.5 \_ \pm 7.8$ mins while in the standard group the duration was $61.8 \pm 11.7 \mathrm{mins}$ and this difference is statistically significant [42].

Analgesics were used in both the groups, comparatively; analgesics were required for more days in the standard group than the tubeless group as only $4.54 \%$ cases in tubeless group required analgesics on $3^{\text {rd }}$ day, while $30.3 \%$ cases in standard group required analgesics on the $3^{\text {rd }}$ day. The difference was found to be statistically significant and our study shows that analgesics were required for $\mathrm{s}$ shorter duration in the tubeless group compared to the standard group. The mean duration of analgesics in the standard group was $2.5 \pm 0.77$ while in the tubeless group was $1.63 \pm 0.56$ and it was found to be statistically significant. In the standard group the pain score was of 4 in most patients while in the tubeless group the pain score was of 2 signifying that there was lesser post-operative pain in the tubeless group compared to the standard group. In study by Suresh Bhat, the analgesic requirement was significantly lower in the tubeless group relative to the standard group [69]. In study by SM Aghamir, the analgesics requirement in the tubeless group was $9.8 \mathrm{mg}$ of morphine on an average while in the other group; it was $28.4 \mathrm{mg}$ which was found to be statistically significant [52]. In study by $\mathrm{H}$ Shah, the postoperative pain score in tubeless group was 4.4 on day 0 and 2.8 on day 1 while in the standard group, it was 7.1 on day 0 and 5.2 on day 1 and the analgesic requirement was less in the tubeless group which was $102.7 \mathrm{mg}$ of diclofenac in the tubeless group while it was $249.9 \mathrm{mg}$ in the standard group. Comparing the difference in the postoperative pain scvore and the dose of analgesics required, the difference was found to be statistically significant [50]. In study by S Dayapule, the average duration of use of analgesics in the standard group was $4.4 \pm 0.9$ days while in the tubeless group it was $2.6 \pm 0.5$ and difference is statistically significant. The VAS pain score in the tubeless group was $2.3 \pm 0.5$ while in the standard group, it was $3.7 \pm 1.1(\mathrm{p}<0.0001)(42)$. In study by Desai et al. in 2004, they performed a prospective randomized study of patients undergoing PCNL with conventional large-bore nephrostomy drainage, smallbore nephrostomy drainage, or no nephrostomy drainage. They concluded that tubeless PCNL was associated with least amount of pain [31].

The hospital stay in the standard group was in the range of 2-7 days in the standard group while it was in the of 1-5 days in the tubeless group. The mean hospital stay in the standard group was $4.42 \pm 1.61$ days while in the tubeless group it was $2.52 \pm 1.05$ days. Comparing the hospital stay in both the groups, the difference was found to be statistically significant. In study by SM Aghamir, the average length of the tubeless group was 1.6 days while in the standard group was 5.2 days and the difference was statistically significant [52]. In study by $\mathrm{H}$ Shah, the hospital stay in the standard group was 56.3 hours while in the tubeless group, it was 33.6 hours and the difference is statistically significant [50]. In study By S Bhat, the duration of hospital stay was 99.84 hours in the standard group while it was 76.8 hours in the tubeless group and this was statistically significant. In study by $\mathrm{S}$ Dayapule, the average hospital stay in the standard group was $5.0 \pm 0.5$ days while in the tubeless group; it was $3.5 \pm 0.8$ days $(\mathrm{p}<0.0001)$. In study by MM Desai, the hospital stay was approx. 3.4 days in the tubeless group while it was 4.4 days in large bore PCNL and it was significant [31]. The most common complication in both the groups was fever. There were two cases of leakage from the wound site in the tubeless group. In the post-operative period, two patients in the standard group and one patient in the tubeless group developed pleural effusion.

\section{CONCLUSIONS}

This research work is carried out over a period of 1.5 years prospectively.

\section{We conclude that}

- Tubeless PCNL is safe and effective methods of renal stone management

- Tubeless PCNL is comparable to the standard 
PCNL in terms of postoperative outcomes and safety and seem to have benefits in terms of length of hospitalization; postoperative pain and all these features might make tubeless PCNL the new standard.

- Early discharge also helps in reducing the financial burden to the patient.

Although prospective and larger-scale studies may be needed to confirm the result of this study, tubeless PCNL may be an alternative for the management of renal stones in selected patients.

\section{REFERENCES}

1. Park S, Pearle MS. Urolithiasis: update on metabolic evaluation of stone formers. Sci World J. 2005; 5:902e14.

2. Moe OW. Kidney stones: pathophysiology and medical management. Lancet; 2006; 367: 333-44.

3. Duvdevani M, Razvi H, Sofer M. Contemporary percutaneous nephrolithotripsy: 1585 procedures in 1338 consecutive patients. J Endourol. 2007; 21: 924-9.

4. Williams SK, Hoenig DM. Synchronous bilateral percutaneous nephrostolithotomy. $J$ Endourol. 2009; 23: 1707-12.

5. Istanbulluoglu MO, Cicek T, Ozturk B, Gonen M, Ozkardes H. Percutaneous nephrolithotomy: nephrostomy or tubeless or totally tubeless? Urology. 2010; 75:1043-6.

6. Paul EM, Marcovich R, Lee BR, Smith AD. Choosing the ideal nephrostomy tube. BJU Int. 2003;92:672-7.

7. Akman T, Binbay M, Yuruk E, Sari E, Seyrek M, Kaba M. Tubeless Procedure is Most Important Factor in Reducing Length of Hospitalization After Percutaneous Nephrolithotomy: Results of Univariable and Multivariable Models. Urology. 2011; 77:299-304.

8. Li H, Zhang Z, Li H, Xing Y, Zhang G, Kong X. Ultrasonography-guided percutaneous nephrolithotomy for the treatment of urolithiasis in patients with scoliosis. Int Surg. 2012;97:182-8.

9. Winfield HN. Weyman P. Clayman RV: Percutaneous nephrostolithotomy: Complications of premature nephrostomy tube removal. J Urol. 1986; 136: 77-9.

10. Ryan PC, Lennon GM, McLean PA, Fitzpatrick JM: The effects of acute and chronic JJ stent placement on upper urinary tract motility and calculus transit. Br J Urol. 1994; 74: 434-9.

11. Crook TJ, Lockyer CR, Keoghane SR, Walmsley BH. Totally tubeless percutaneous nephrolithotomy. J Endourol. 2008; 22: 267-271.

12. Akin Y, Basara I, Yucel S, Gulmez H, Ates M, Bozkurt A. Is tubeless percutaneous nephrolithotomy really less injurious than standard in the midterm? J Endourol. 2013; 27:1192-6.

13. Wickham JE, Miller RA, Kellett MJ, Payne SR. Percutaneous nephrolithotomy: One stage or two.
Br JUrol. 1984;56:582-5.

14. Bellman GC, Davidoff R, Candela J, Gerspach J, Kurtz S, Stout L. Tubeless percutaneous renal surgery. J Urol. 1997;157:1578-82.

15. Candela J, Davidoff R, Gerspach J, Bellman GC. Tubeless" percutaneous surgery: A new advance in the technique of percutaneous renal surgery. Tech Urol. 1997;3:6-11.

16. Al-Ba'adani HT, Al-Kohlany KM, Al-Adimi A. Tubeless percutaneous neprolithotomy: the new gold standard. Int Urol Nephrol 2008; 40: 603-8.

17. Sivalingam S, Cannon ST, Nakada SY. Current practices in percutaneous nephrolithotomy among endourologists. J Endourol 2014; 28:524-7.

18. Rifaioglu MM, Onem K, Buldu I, Karatag T, Istanbulluoglu MO. Tubeless percutaneous nephrolithotomy: Yes but when? A multicentre retrospective cohort study. Urolithiasis. 2014;42: 255-62.

19. Zilberman DE, Lipkin ME, de la Rosette JJ. Tubeless percutaneous nephrolithotomydthe new standard of care? J Urol. 2010; 184:1261-66.

20. Shoma AM, Elshal AM. Nephrostomy tube placement after percutaneous nephrolithotomy: critical evaluation through a prospective randomized study. Urology. 2012; 79:771-6.

21. Agrawal MS, Agrawal M. Tubeless percutaneous nephrolithotomy. Indian J Urol. 2010; 26(1): 1624.

22. Ramsay JW, Payne SR, Gosling PT, Whitfield HN, Wickham JE, Levison DA. The effects of double J stenting on unobstructed ureters. An experimental and clinical study. Br J Urol. 1985;57:630-4.

23. Rupel E, Brown R. Nephroscopy with removal of stone following nephrostomy for obstructive calculus anuria. J Urol. 1941;46: 177-79.

24. Fernstrom I, Johannson B. Percutaneous nephrolithotomy: a new extraction technique. Scand J Urol Nephrol. 1976;10: 257-61.

25. Agrawal MS, Agrawal M, Gupta A, Bansal S, Yadav A, Goyal J. A randomized comparison of tubeless and standard percutaneous nephrolithotomy. J Endourol. 2008;22:439-42.

26. Jackman SV, Hedican SP, Peters CA, Docimo SG. Percutaneous nephrolithotomy in infants and preschool age children: experience with a new technique. Urology. 1998;52:697-701.

27. Kader AK, Finelli A, Honey RJ. Nephroureterostomy-drained percutaneous nephrolithotomy: modification combining safety with decreased morbidity. J Endourol. 2004;18:2932.

28. Li LY, Gao X, Yang M, Li JF, Zhang HB, Xu WF. Does a smaller tract in percutaneous nephrolithotomy contribute to less invasiveness? A prospective comparative study. Urology. 2010;75:56-61

29. Aghamir SM, Hosseini SR, Gooran S: Totally tubeless percutaneous nephrolithotomy. $J$ Endourol. 2004; 18: 647-8. 
30. Limb J, Bellman GC: Tubeless percutaneous renal surgery: review of first 112 patients. Urology. 2002; 59: 527-31.

31. Desai MR, Kukreja RA, Desai MM. A prospective randomized comparison of type of nephrostomy drainage following percutaneous nephrostolithotomy: large bore versus small bore versus tubeless. J Urol. 2004; 172: 565-7.

32. Marcovich R, Jacobson AI, Singh J. No panecea for drainage after percutaneous nephrolithotomy. $J$ Endourol. 2004; 18: 743-7.

33. Feng MI, Tamaddon K, Mikhail A, Kaptein JS, Bellman GC. Prospective randomized study of various techniques of percutaneous nephrolithotomy. Urology. 2001; 58: 345-50.

34. Kirac M, Tepeler A, Bozkurt OF, et al. The efficacy of bupivacaine infiltration on the nephrostomy tract in tubeless and standard percutaneous nephrolithotomy: a prospective, randomized, multicenter study. Urology. 2013;82:526-31.

35. Zhong Q, Zheng C, Mo J, Piao Y, Zhou Y, Jiang Q. Total tubeless versus standard percutaneous nephrolithotomy: a meta-analysis. $J$ Endourol. 2013;27:420-26.

36. Holman E, Khan AM, Pasztor I. Simultaneous bilateral compared with unilateral percutaneous nephrolithotomy. BJU Int. 2002;89:334-8.

37. Silverstein AD, Terranova SA, Auge BK. Bilateral renal calculi: assessment of staged $\mathrm{v}$ synchronous percutaneous nephrolithotomy. $J$ Endourol. 2004; 18:145-51.

38. Karami H, Jabbari M, Arbab AH. Tubeless percutaneous nephrolithotomy: 5 years of experience in 201 patients. J Endourol. 2007; 21: 1411-3.

39. Lai WH, Jou YC, Cheng MC. Tubeless percutaneous nephrolithotomy: Experience of 1000 cases at a single institute. Urological Science. 2017; 28: 23-6.

40. Jou YC, Cheng MC, Lin CT, Chen PC, Shen JH. Nephrostomy tube-free percutaneous nephrolithotomy for patients with large stones and staghorn stones. Urology. 2006;67:30-4.

41. Shah HN, Sodha HS, Khandkar AA, Kharodawala S, Hegde SS, Bansal MB: A randomized trial evaluating type of nephrostomy drainage after percutaneous nephrolithotomy: small bore $\mathrm{v}$ tubeless. J Endourol. 2008; 22: 1433-9.

42. Dayapule S, Vaddi S, Bhaskar GV, Pathapati R. Efficacy and Safety of Tubeless Percutaneous Nephrolithotomy versus Standard Percutaneous Nephrolithotomy. IJSS Journal of Surgery. 2016;2(4):16-20.

43. Khan N, Khan R. Tubed vs tubeless PCNL, our experience at North West General Hospital and Research Center, Peshawar. Pak J Surg. 2015; 31(3):17-23.

44. Falahatkar S, Khosropanah I, Roshani A, Neiroomand H, Nikpour S, Nadjafi-Semnani M.
Tubeless percutaneous nephrolithotomy for staghorn stones. J Endourol. 2008;22: 1447-51.

45. Xun T. Tubeless and standard percutaneous nephrolithotomy in elderly patients. BMC Urology. 2017; 17:102-11.

46. Kumar S, Singh S, Singh P. Day care PNL using 'Santosh-PGI hemostatic seal' versus standard PNL: a randomized controlled study. Cent European J Urol. 2016;69:190-7.

47. Kara C, Resorlu B, Bayindir M1. A randomized comparison of totally tubeless and standard percutaneous nephrolithotomy in elderly patients. Urology. 2010; 76:289-93.

48. Proietti S, Knoll T, Giusti G. Contemporary ureteroscopic management of renal stones. Int $J$ Surg. 2016;36:681-7.

49. Kwon S, Kim HG. A comparative study between standard and tubeless percutaneous nephrolithotomy. Korean J Urol. 2007;48: 45-8.

50. Shah H, Khandkar Al. Tubeless percutaneous nephrolithotomy: 3 years of experience with 454 patients. Eur Urol Supp. 2008;7:190-9.

51. Karami H, Gholamrezaie HR. Totally tubeless percutaneous nephrolithotomy in selected patients. J Endourol 2004;18:475-6.

52. Aghamir SM, Mohammadi A, Mosavibahar SH, Meysamie AP. Totally tubeless percutaneous nephrolithotomy in renal anomalies. J Endourol. 2008; 22: 2131-34.

53. Aghamir SM, Modaresi SS, Aloosh M. Totally tubeless percutaneous nephrolithotomy for upper pole renal stone using subcostal access. J Endourol. 2011; 25(4):583-86.

54. Yang RM, Bellman GC. Tubeless percutaneous renal surgery in obese patients. Urology. 2004; 63: 1036-40.

55. Bilen CY, Gunay M, Ozden E, Inci K, Sarikaya S, Tekgul S. Tubeless mini percutaneous nephrolithotomy in infants and preschool children: a preliminary report. J Urol. 2010; 184: 2498-502

56. Shah HN, Mahajan AP, Hegde SS, Bansal M. Tubeless percutaneous nephrolithotomy in patients with previous ipsilateral open surgery: a feasibility study with review of the literature. J Endourol. 2008; 22: 19-24.

57. Sofikerim M, Demirci D, Huri E, Ersekerci E, Karacagil M. Tubeless percutaneous nephrolithotomy: safe even in supracostal access. $J$ Endourol. 2007; 21: 967-71.

58. Lee $\mathrm{SC}$, Kim $\mathrm{CH}$, Kim KT. Is tubeless percutaneous nephrolithotomy a feasible technique for the treatment of staghorn calculi? Korean $J$ Urol. 2013; 54: 693-6.

59. Shpall AI, Parekh AR, Bellman GC. Modification of tubeless percutaneous nephrolithotomy: antegrade stent with flank tether. Urology. 2006; 68: $880-2$

60. Abou-Elela A, Emran A, Mohsen MA, Reyad I, Bedair AS, Kader MA. Safety and efficacy of tubeless percutaneous renal surgery. J Endourol. 
2007; 21: 977-84.

61. Michel MS, Trojan L, Rassweiler JJ. Complications in percutaneous nephrolithotomy. Eur Urol. 2007; 51: 899-906.

62. Shah HN, Hegde S, Shah JN, Mohile PD, Yuvaraja TB, Bansal MB. A prospective, randomized trial evaluating the safety and efficacy of fibrin sealant in tubeless percutaneous nephrolithotomy. $J$ Urol. 2006; 176: 2488-92.

63. Jou YC, Cheng MC, Sheen JH, Lin CT, Chen PC. Cauterization of access tract for nephrostomy tubefree percutaneous nephrolithotomy. J Endourol. 2004; 18: 547-9.

64. Leibovici D, Cooper A, Lindner A. Ureteral stents: morbidity and impact on quality of life. IMAJ Isr Med Assoc J. 2005; 7: 491-4.

65. Yew J, Bellman G. Modified "tubeless" percutaneous nephro-lithotomy using a tail stent. Urology. 2003;62:346-9.

66. Shpall AI, Pa re kh AR, Bellman GC. Tubeless Percutaneous Nephrolithotomy with Antegrade Stent Tether: Clinical Experience. J Endourol.
2007;21:973-6.

67. Berkman DS, Lee MW, Landman J, Gupta M. Tubeless Percutaneous Nephrolithotomy (PCNL) with Reversed Polaris(trade mark) Loop Stent: Reduced Postoperative Pain and Narcotic Use. $J$ Endourol. 2008;22:2245-50.

68. Singh I, Kumar A, Kumar P. Ambulatory PCNL" (tubeless PCNL under regional anesthesia): A preliminary report of 10 cases. Int Urol Nephrol. 2005;37:35-7.

69. A Tyagi, B Ramana, D Ramesh, J Jayaraju, P Prasad, S Suresh Goud. A prospective study on outcomes of tubeless percutaneous nephrolithotomy - our experience in SVIMS. International Journal of Contemporary Medical Research 2016;3(9):2608-2611.

70. Bhat S, Lal J, Paul F. A randomized controlled study comparing the standard, tubeless, and totally tubeless percutaneous nephrolithotomy procedures for renal stones from a tertiary care hospital. Indian J Urol. 2017;33:310-4. 